\title{
Morphological Categorization and its Role in Design Method
}

\section{Joon Bum Kwun and HeeJoon Whang}

Department of Architecture, Sejong University, Seoul, Korea

Department of Architecture, Hanyang University, Seoul, Korea

\begin{abstract}
The first attempt in architectural design theory to consider the perceptual and metaphysical dimension separately with a fully modern scientific manner, which exist in contrast to the Renaissance idea, was Claude Perrault, who emphasized the cognitive factors as an important scientific human issue in building design in 1683 in his book "Ordonnance". Even today many elaborated works to reveal the mysterious design processes based on a set of rational approaches have been introduced since the Design Method movement in 1960's. Their pioneering and challenging efforts to rationalize design process have been mostly rely on the cultural issues whether it takes a qualitative or quantitative stance. On the other hand, however, today's computer generated free form architecture seems not to be aware of those lessons learned from the past and, therefore, this study conducted an extensive research through exploring on morphological building forms with cultural issues to fill up the missing and/or lacking characteristics of today trend in building design.
\end{abstract}

Keywords: Morphology, Labeling, Categorization, Design Method

\section{INTRODUCTION}

\subsection{Background}

It is certain that architects will need to have adaptive aesthetic skill for a better arbitrary manipulation of forms, although it does not necessary have to resemble the form or style of the past as in the case of Postmodernism. However, despite of recent trend of free form design, architects will still have to consider about various different building forms according to the functional requirements of the building task. Firstly, architects will have to be aware of what the relationship is between the building and the people who use it, which is both the prerequisites and effect of architecture. According to Christian Norberg Schulz (1965), however, we still don't have an answer to the problem of whether the differentiation should also acquire a symbolizing aspect by the assignment of particular forms to particular functions when the purpose is to represent a cultural structure and this half century old argument still remains and applies even today. Therefore, this study will explore the role of the pre-conceived notion of form in the design process as an important method that affects architect's decision making on the formation of contemporary architecture.

Corresponding Author: Kwun, Joon Bum, Associate Professor Department of Architecture, Sejong University 98 Kunja-Dong, Kwangjin-Ku, Seoul, 143-747, Korea Tel: +82 234083834 e-mail : joonkwun@sejong.ac.kr

This is an Open Access article distributed under the terms of the Creative Commons Attribution Non-Commercial License (http://creativecommons. org/licenses/by-nc/3.0/) which permits unrestricted non-commercial use, distribution, and reproduction in any medium, provided the original work is properly cited.

\subsection{Objective, Hypotheses and Significant of The Study}

The objective of this study is not to introduce a new theory regarding morphological notion in architectural form or design process in general. However, it seems that it would be necessary to provide a conservative stance and precaution against to today's cultureless computer generated free form architecture. Therefore, the problematic view and the hypotheses of this study is that today's design process, in general, trends toward simplicity based on digital information technology (i.e. CAD, BIM, Rhino etc.), whereas architects desire complexity based on strong visual dynamics and multifarious sequences that does not deliver its cultural or symbolic meaning.

The significant of this study is to collect and review a wide range of contemporary theories that has stressed up the role of morphological form, which has gained common cultural sense and cognitive intentions. Consequently, this study hopefully provide an opportunity to re-evaluate the missing and lacking aspects of today's design trend, which is the extreme opposite of the Modern and Postmodern architecture in the sense of culture based morphological notion.

\section{THE ROLE OF MORPHOLOGY}

In general, people tend to group buildings into categories according to their functional purpose (Tversky, 1977), which we labels as house, school and etc. (Tversky \& Hemenway, 1983). Therefore, it is essential to create categories in order to understand the various and diverse incoming information and it seems that the public already have a cognitive image of any known object, which is overlapped with many individual memories. Also, Kevin Lynch (1960) argues that perhaps people have series of images and those images are very important for a successful interaction 
with the environment and the cooperation with his or her fellows. Moreover, a large number of studies (Alexander 1977, Aymonino 1985, Berman 1988, Galle 1999, Haber 1968, Hochberg and Brooks 1962) have shown that the realist images in social science are influenced by the way we understand its structure based on its physical identity.

In our normal experience, there exist a percept, a concept and a representation (Layder, 1990). Meanwhile, there is no doubt that the general public linguistically classifies objects. Triandis (1972) provided insight into the cultural ramification of very complex stimuli, as architectural form certainly is. He describes in general terms the usefulness of examining limited verbal categories when attempting to understand complex stimulation.

Likewise, in architecture, one does see a building and interprets it, usually putting that experience into words (Jencks, 1984), which can provide the basis for a morphological categorization. For instance, most of us will have had an experience with building called office, church, etc. Common visual characteristics make it possible to put similar buildings into words although they may have different shape. Smithies (1981) also considered architectural labeling to be a direct function of the collective characteristics of building in an identifiable form. Similarly, the division of buildings into particular groups will depend on what the building is expected to do. In other words, the building tasks, what kinds of part will the designs have, and what visual property is considered.

The main point in this discussion so far is the value of having the cognitive notion of building can be used to reference and to make an appropriate choice for a future design project. Glucksburg and Danks (1968) supported evidence to this simple idea that object classification into architectural forms affects the response general public, not only in the focus and remembering of the object, but also object usage as well.

\section{THE NATURE OF DESIGN PROCESS}

One study shows that most of the architects in the relationship with their clients, preferred to be interact with each other from the very beginning of the project (Lawson, 1997). Similarly, it is assumed that client will indicate to the architect his or her preliminary idea of the building desired, what function it should provide, and what characteristics the architect should consider in its design. However, the above study claims that most architects never get a brief from a client that can form the basis of their design. Even if the architect have been informed by the client, obviously, it would often be easier for the client to present their problems by citing existing or past solutions familiar to them. This could be very interesting and might remain as one of the many characteristics of design process, which could be valuable to study.

In our daily experience, we observe different ways of design thinking and claim that imagining based on reasoning is perhaps the most important attitude for the architects. Teleology and methodology towards a specific design conclusion could be considered as the realm of reasoning, which requires logical problem solving ability. Imagining, on the other hand, suggests more approach liberal through intuitive and creative inspiration stemming from an individual personality or on personal experience. While pure art, which is mostly conscious of imagining, architectural design gives its value for solving a real world problem that rational evaluation is required. Appropriately combining and controlling these two different thoughts will necessarily result in good design. Research conducted by Kneller (1965) identifies up to five stages of design process, which are 'first insight,' 'preparation, 'incubation', illumination', and 'verification' (Figure 1).

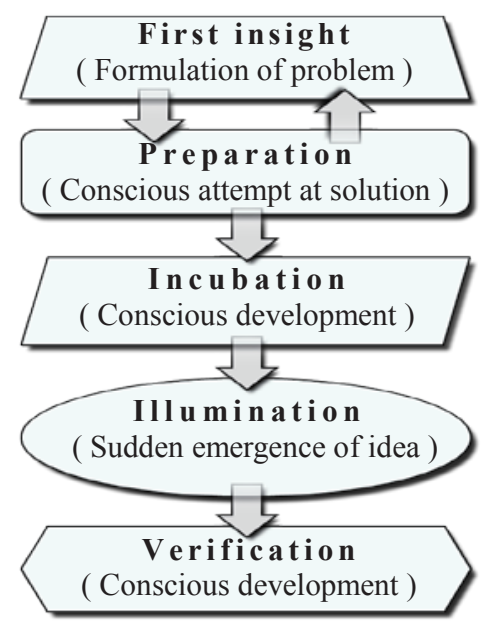

Figure 1. Five phases in the creative process by Kneller

At 'first insight' stage, architect simply needs to be aware of the existing problem. Thus the problem situation has been formulated and it needs to prepare a scheme to solve the problem. On the second stage, 'preparation', architect starts to seek for a problem solution and it can be seen on the diagram that repeated work, between this stage and the first will take time to get a design clue. The third stage, 'incubation', usually requires no conscious effort because it will depend totally on imagination or inspiration. Nevertheless, the length of this period can vary according to an architect's experience. The next stage, 'illumination', can be considered together with 'incubation' stage. It is not certain how, why and even when the human mind suddenly comes up with an idea, but some argue that in this period architects maintain a continuous screening process of all the data, which was collected during the intensive earlier periods. Finally, at the 'verification' stage, the concept will be evaluated, tested and elaborated. If it seems that there is any flaw with the idea, the process can be reformulated and a new period of investigation begun, and so on.

Another ambitiously programmed design method was developed by Geoffrey Broadbent (1973) specifically for use in architecture but, it actually has many generic qualities. In reality Broadbent's method probably does not hold together as a totality since it relies upon four distinct ways of generating design form which he called the 'pragmatic', 'iconic', 'canonic', and 'analogical' methods. Broadbent arrived at this taxonomy from a study of the history of architecture and shows how each of his four techniques have been used at various times. Broadbent suggests a complete design method could find the designers using all four of his tactics in an ordered and organized way, finally selecting from amongst the solutions produced. There is no evidence that designers actually work like this, but his four tactics are worthy of study and form a very useful addition to the designer's tool kit of tactics for controlling design thought. 
Pragmatic design is simply the use of available materials and methods of construction, generally without innovation, using standard components as though selecting from a catalog. Provided the designer has a good grasp of the strengths and weaknesses of traditional and established techniques this method certainly has it uses. It is essentially traditional and conservative and, therefore, an approach involving low risk, unlikely to lead to dramatic failures. This is virtually a pattern book approach and unlikely to yield impressive design or move design ideas forward. However, it may well prove a valuable tactic in identifying a range of possible forms for a design or parts of a design.

Iconic design is even more conservative in that it effectively calls for the designer to copy existing solutions or icons. Speculative house builders seem to work this way by reproducing their standard house types irrespective of local conditions or the external constrains of the site. While this is unlikely to appeal to the creative mind, such an approach does have its value and supporters. Ladsun (1965) in his article "An architect's approach to architecture", has been critical of architects for beginning their design process with a blank sheet of paper as if each problem were entirely new. By using iconic techniques designers might begin with existing solution and modify them to meet the new conditions. In other words, in Iconic design, the designer starts with some fixed "mental images" of a familiar building form, and relates the constancy of building form to the constancy of building crafts and to its relation in turn to the cultural consciousness. This might lead to a greater stability and avoid the commonly found errors in which designers miss the clever way in which vernacular designs solved problems.

Canonic design relies on the use of rules such as planning grids, proportioning systems and the like. Classical architectural styles and their Renaissance successors offered opportunities for such an approach, and we have already seen how Vitruvius and later Alberti laid down such rules. More recently Le Corbusier's 'modulor' can be seen as an attempt to produce canonical rules that allowed for more iconic design. Even more recently, system building techniques relying on modular coordination and standard components have typically generated rather dull results.

Analogical design results from the designer using analogies with other fields or contexts to create a new way of structuring the problem. In other words, in analogic design, visual and formal analogies are usually drawn with existing buildings or sometimes form from nature. Certainly, there are clear examples of significant use of analogical thought in design. Examples would be John Utzon's Sidney Opera House, which idea came from the concept of sail boats and the TWA Terminal at New York, where the idea came from bird. According to Ungers (1982):

If the process of design starts with a conceptual image that forms the basic principle around which the whole is organized, then it is possible to develop, within this image, the full range of fantasy. Designing with conceptual images makes it possible to move from pragmatic to creative thought, from the metric space of numbers to the visionary space of coherent system. (p.23)

The use of organic forms in architecture offers ways of generating aesthetical and also efficient structures with natural characteristics.
Analogies may be used to give integrity to ways of constructing parts of design solutions (Slife, 1995). Broadbent himself seems to suggest that the 'analogical' method is the most promising of these four form generation tactics. However, the trap in this kind of approach is that it may seem a little naïve or even ridiculous, but there is considerable evidence that this process is quite widely used with positive results for some designers.

Many architects dislike the idea of generating alternative designs and in particular to showing various alternatives to clients. This seems very much a matter of personal design style and client management, but leads to a fear among designers that a client may want to pick ideas from several alternatives that maybe impossible or extremely difficult to combine, or that will lead to a solution that lacks in "integrity".

After all, the different design professions are divided not by the kinds of problem they tackle, but by the kinds of object they create. Even with a single design field such as architecture we tend to consider of a project by the building labeling which it is expected will result, such as office, school, house, and so on so forth.

Here again we see the way these guiding principles have been formed an extended period of time in practicing design. There is clearly a two way process. On the one hand the guiding principles influence and set the mental context for each design process. On the other hand, each design problem enables the designer to learn more about guiding principles and express them ever more clearly, eventually resulting in books and lectures. In this sense, design process is also a form of research; it offers an action-based method of advancing knowledge.

\section{ARCHITECTURAL DESIGN METHOD}

In seeking a guiding method for architectural design, it is necessary to identify and understand the real design problems in outlining meaningful solutions. Unfortunately, however, architects or designers are not scholars or philosophers, and therefore, to understand the nature of architectural design, we have to rely on design researchers.

Interest in design method emerged during the 1960s and early 70 s. This was not a technologically oriented form of architectural research, but rather a form of research based on the social and behavioral science studying about the interaction of people with the building environment (Francestcato, 1994). However, in the beginning stage of this field, design method, it represented a mechanical linear process of design creativity. Christopher Jones, a leading advocate of the application of rational design method, can be considered as one who represents the linear process of creativity. According to Jones (1992), many design theorists imply that the most valuable part of the design process is that which goes on inside the practitioner's head, partly out of reach of his or her conscious control.

Jones (1992) argues that most design methods are concerned with externalized thinking and are, therefore, based on rational rather than on mystical assumptions. Figure 2 shows the rational, or systematic, designer to be very much like a human computer, a person who operates only on the information that is fed to him, and who follows through a planned sequence of analytical, synthetic and evaluative steps and cycles until he recognizes the best of all possible solutions. 


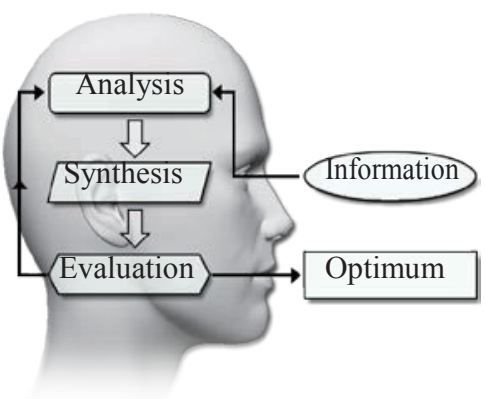

Figure 2. Linear Design Process by Jones.

This kind of linear process for optimization, however, is impossible because the human mind has a limited cognitive capacity. One of the greatest psychologist William James (1892) argues that the "sum total of our impressions never enters into our experience." Therefore, if a practitioner has unlimited time, budget and cognitive capacity, this theory could be possible. However, the problem with the linear design concept is that it is based on a mechanical processing concept, which lacks of human sense. This fact could be the reason why the 'design method' concept does not work. Moreover, if we assume that this kind of theory really works, then the concept of 'voluntary attention' by William James would no longer be necessary.

As Kelbaugh (1996) argues, unconstrained freedom is anathema to designers who need limits just as much as civilization itself needs rules, tradition and conventions. Similarly, Pena (1987) argues that creativity thrives when the limits of a problem are known. The theories of the mind that explain how human creativity works understand that creativity only occur within certain established boundaries and limits.

In this sense, in the field of architecture, the concept of limit that makes creativity possible can be expanded with the morphological catergorization. In other words, categorization of architectural form can expand a practitioner's cognitive capability, which would be helpful in the act of creation. Similarly, the notion of Gestalt pervades in the theories developed within problem solving and information theory. In artificial intelligence and related fields, the notion of pre-conceived morphology pervades in concepts like 'knowledge structure' or 'pattern of thoughts'.

Another reason why the linear process cannot work is because every individual has his or her own unique 'experience. This fact is important because categories of experience (i.e., different events, objects, and places, etc.) can be associated to build a cognitive map. This cognitive map cannot be transferred from one person to another because every human has a different experience and, therefore, becomes an issue. However, morphological labeling in architecture can be considered as a visual cognitive map. This means that pre-conceived formal notion can provide the practitioner with a much needed series of cognitive reference points in order to increase understanding of the design problem through the use of mental, physical or visual simulations. Thereby, formal labeling can enter into the creative act of architectural design in two ways. First, it can be helpful to expand the architect's limited cognitive capacity, and second, it can be used as an effective communication tool.

Architectural design method is the study of the principles, practices and procedures of design, whose main concern is how design 'is' and 'might be' conducted (Cross, 1984). During the past few decades, design theorists (i.e. Broadbent, Cross, Simon, Heath, etc.) proposed a structural framework of the design process, an approach to design problems, and other various aspects of problem solving methodology. In other words, the study of design method includes: the establishment of appropriate structures for the design process; the development and application of new design methods, techniques, and procedures; and some reflection on the nature and extent of design knowledge and its application to design problems (Cross, 1984). Design theories have always been influential in architecture because a single theory cannot build up a monopoly over other aspects of building (Blau, 1993). Furthermore, architects have increasingly turned to design theory during the last several decades because the bridge between planning and architecture has significantly degenerated since the Second World War.

\section{MORPHOLOGICAL APPROACH IN DESIGN METHOD}

A morphological outcome in architecture is the result of many factors. For example, Symes (1994) discussed pre-conceived formal thinking in professional work as encompassing three particular components of design practice:

1. The adoption of a design method or approach, which provides a way of defining the architect's relationship to a professional context;

2. The use of spatial variations, which provide a vehicle for the storage and transmission of architectural knowledge and experience;

3. The use of functional categories, which provide a framework for the modification of user needs.

Similarly, Robinson (1994) argues that architectural categorization offers several ways to develop perspectives on a design process:

1. Understanding the nature of existing forms;

2. Understanding how different forms may need to be adjusted when they are applied to particular circumstances;

3. Developing design possibilities by identifying individual attributes of existing forms that may be recombined in innovative ways.

Likewise, most of the morphological research concerning architectural design methods can be narrowed down to three major objectives. These objectives include reviewing architectural forms in terms of:

1. Tradition: Iconic analysis of past architectural building forms. In this case, formal morphology has the effect of forming typicality in category membership (Rosch, 1978).

2. Adaptation: Analytical variation in dealing with architectural building forms. In this case, morphology is noticeable and may consist of typical instances, functional parameters, or the ideal exemplars of the category (Smithies, 1981). 
3. Structure: Modifying a building's function, meaning, etc., pertaining to the design method. In this case, architectural morphology is a synthesis of overall exemplars (Jansson, Condoor \& Brock, 1993).

In this section, the role of architectural formal categorization in the design method will be explored based on these three major views.

\subsection{Traditional Aspect of Form Making}

The traditional aspect of architectural formal categorization as a design method deals with an abstract model and, in this case, the notion of form usually remains in the systematic classification stage between the specific object and the categorization class. During the 1960s, a systematic design approach began to emerge in all of the different professional specialist areas of design (Cross, 1984). It is not difficult to find scholarly evidence to support this idea, and in most cases classified buildings share homogeneous characteristics in their functional aspects. Norman (1993) tackles the analysis of the communication between building and the public exclusively with regard to the functional aspects of an object, which means that the division of buildings into particular categories will depend on what the building is expected to mean. Lynch (1998) and Mitchell (1990) anticipated this idea by arguing that perhaps people have a series of images, and those images are very important to their successful interaction with the environment. Moreover, a significant number of studies have shown that our memory of the environment is influenced by the way we categorize its components (e.g. Tversky \& Hemenway, 1983) and again, according to Lynch (1960), the 'imageability' of the environment depends both upon its identity and its physical structure.

As with the 18th century theorist Durand, this is involves pattern book approach and is, therefore, essentially traditional and conservative while avoiding the danger of serious design failure. Symbolized form is the literal repetition of a tried and accepted building shape. Therefore, it is even more traditional and conservative because it guides architects to copy existing solutions and morphological icons.

Similarly, despite a considerable amount of research on architectural formal language and design methods, many studies confirm that there is a significant difference between architects and the public in building preferences and significance (Devlin \& Nasar, 1989; Groat, 1982; Morris, 1982). This is because even though the morphological terms are culturally shared, preferences may not be identical. Robinson (1994) addresses this problem by exploring the different priorities of the architect and the public. She argues that when the public thinks about a basic form, such as a house, they may be cued by a formal icon which generally represents a set of human issues first, and physical forms second. For an architect, however, the related shape is first presented as a set of physical building forms and considers the human issues second. The following examples by Jack Nasar (1998) show the disagreement about building preferences and meaning between architects and non-architects (Figures 3 ).

Therefore, it is important to understand the morphological categories in order to understand the various and diverse forms of incoming information. It seems that the public already has an image formed of any known object, and this image is overlapped by many individual architectural categorizations (Posner, 1973).
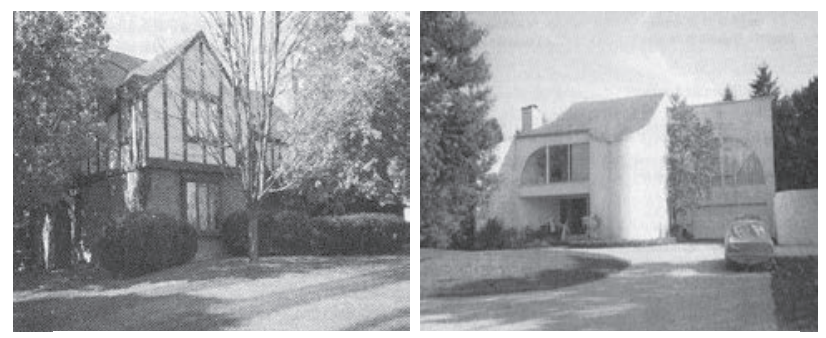

Figure 3. A house form most liked by the public (left) and a house form most liked by architects (right)

\subsection{Adaptational Aspect of Morphology}

The adaptational aspect of architectural pre-conceived notion as a design method does not refer to the form itself as an essence, and the physical components are often more heterogeneous than in the traditional approach. It emphasizes the adaptive aesthetic skill of better building forms and, therefore, does not necessarily intend to resemble the building form or components of the past (Rasmussen, 1959; Rowe, 1987; Sherwood, 1978).

However, in considering heterogeneous morphological forms, the concept of 'familiarity' still plays an important role. Robinson (1994) argues that the most important reason why the public does not readily understand or accept the innovative architectural outcome is because the resulting building looks unfamiliar. Also, according to Kaplan and Kaplan (1981), familiarity not only fosters compactness, but it also leads to a coherence that significantly increases the capacity for manipulation of the item in question. Familiarity provides ways for facilitating the basic process of searching for a solution. In this regard, pre-conceived architectural morphology can be considered to have a direct effect on the collective characteristics of the building as an identifiable form (Smithies, 1981). Ungers (1982) argues that when the design process starts with a conceptual image that supplies the basic principle around which the whole is organized, then it is possible to develop a project based on this image. In other words, designing with conceptual images makes it possible to move from pragmatic to creative thought.

Also, Tice (1993) confirms that architectural formal categorization is an effective tool for building both analysis and creation. His main assumption regarding maximizing the effectiveness of building forms is that morphological studies offer an effective design framework for change, and that this change must be shown to be a transformation from some known state. The ability to conceive the transformation of familiar form is essential if the interpretative nature of analysis and the dynamics of the design process are to be fully engaged. Schön (1988) described categorical image as a range of already interpreted information about the detailed implications of possible design actions, that offer a shortcut in producing building forms, explaining ideas to clients, and personally reassuring architects themselves when they face risks associated with innovation. Similarly, Broadbent (1973) argues that simply copying existing architectural forms without innovation will not guarantee a good design because it may be difficult to adapt an icon that is copied literally to a different site condition and situation. However, he regards the iconic design method as a 
useful technique if architects begin with existing solutions or icons and then adapt them to new architectural conditions, as in the case of the new Tokyo City Hall Complex Building designed by Kenzo Tange (Figure 4).
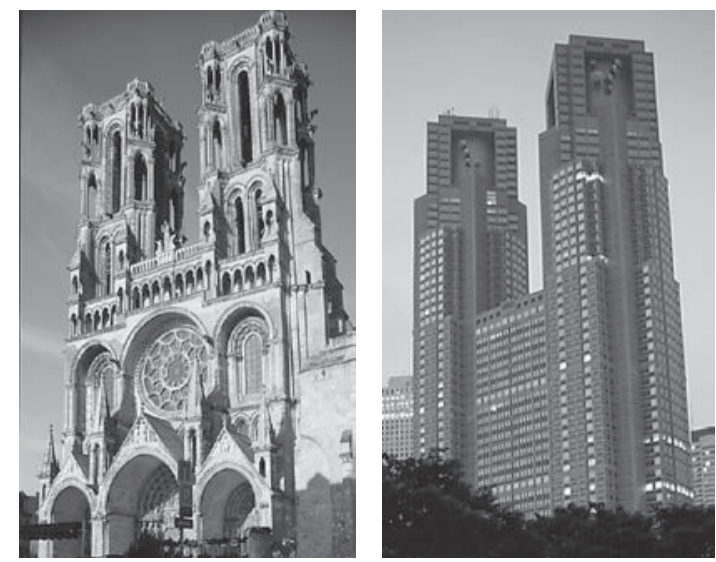

Figure 4. Morphological similarities between Laon Cathedral in France and Tokyo City Hall by Kenzo Tange

The City Hall's building image represents the adaptive form of a French Gothic Cathedral and it transforms the symbolic meaning of the Medieval with its magnificent atmosphere, to an important core building in the modern city. With this method, it can be understood that architects begin with some fixed image of a familiar building shape and connect the constancy of the building form to the cultural consciousness.

This adaptive iconic approach can be applied not only to a visual image, but also to plan form which can be equally iconic as, for example, the work of architects who generally follow the plan form (icon) of Palladio (e.g. Frank Lloyd Wright, Alvar Aalto, et al). Le Corbusier's house design also retains the same public-private spatial composition as a traditional notion of a house. As such, the basic architectural morphology provides a locus for improvement of the fit between cultural ideals and design environments (Robinson, 1994). Groat (1983) emphasizes the interpretation of artifacts within the context for which they were made. She argues that the making or interpretation of contemporary architecture involves not only current conventions and empirical knowledge, but should also include an attempt to recall and reexamine the intellectual and formal conventions internal to architecture throughout history.

\subsection{Structural Aspect of Morphology}

The structural aspect of architectural formal categorization as a design method often includes the typical concept of a building's character, and represents the restructured form of the past building's image. According to Gelernter (1995), all art forms are first created within universal objective design elements and compositional principles; only later do outside constraints and technological possibilities modify these art forms. It seems that architects are most interested in this structural approach.

The restructuring of organic types into architecture may offer ways of generating aesthetically appropriate building forms, as well as efficient structures with natural characteristics. As in the case of Pietiläs concept of 'literal morphology' in which the Malmi church (Figure 5) is designed to actually resemble a rock formation
(Quantrill, 1985), the analogical design method may be used to give integrity to new ways of constructing design solutions.

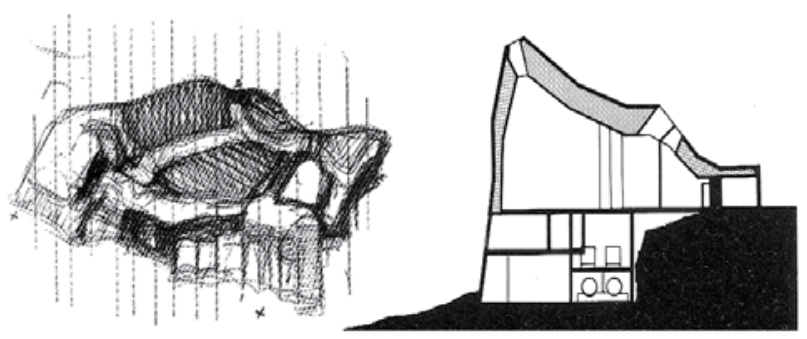

Figure 5. The Malmi Church that resembles a rock formation by the Finnish Architect Reima Pietilä

However, the most serious negative aspect of using organic forms in architecture is that the concept may seem a little naive, ridiculous, or, even more importantly, the building form may not able to deliver its meaning to the public. It also has the potential to create a completely new building form, which runs a risk of being unrecognizable because the public may not find it sufficiently familiar. Thus, if the architects have a good comprehension of the role of architecturally pre-conceived formal notion, it will reduce the danger of excessive deviation from already tested building forms (Francescato, 1994).

Furthermore, restructuring accepted architectural formal language instead of organic forms in building design would be more promising and secure more positive results for most architects, as in the case of the Kaleva Church (Figure 6) designed by Pietilä. This design can be seen as an experiment in a convexconcave morphology, which is a modern attempt to stay within the spirit of the gothic and the baroque without their stylistic limitations (Quantrill, 1985).
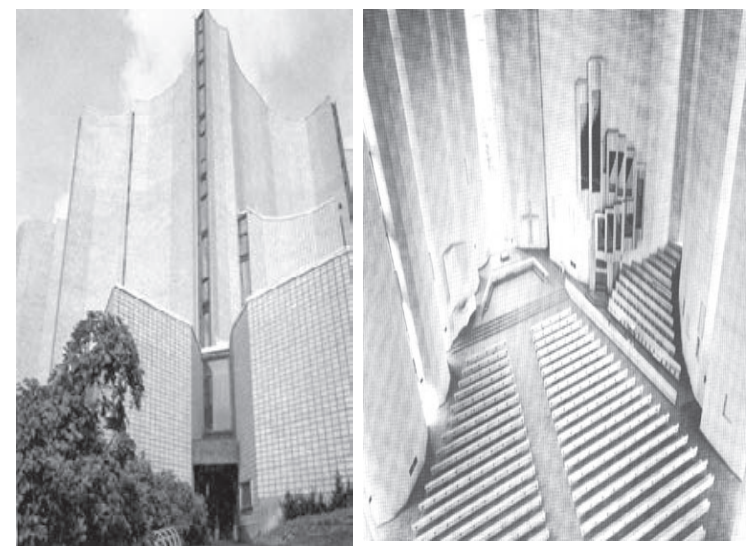

Figure 6. Exterior and Interior of the Kaleva Church that resembles the Gothic and the Baroque Architecture

\section{DISCUSSION}

Ladsun (1965) has criticized architects for design processes that emerge from out of nowhere, as if each problem was entirely new. This seems very much a matter of an architect's personal design approach that selects design ideas from several different alternatives that may be impossible or extremely difficult to combine, or that might lead to a solution that lacks integrity. Ladsun's criticism, 
which is nearly a half century old argument, still and even more applies today because the modern society's digital information technology provides more multi cultural experiences than any other generations before. This trend also provides all new extended cognitive perceptions on various domains. As a result, these circumstances created a new sensual balance on manmade environment with, so called, freeform architecture that, not only lacks in cultural integrity, but also simply screams out for meaningless attention.

However, this study collected and reviewed a wide range of contemporary theories that stressed up the morphological forms with cultural integrity to emphasize the importance of common cultural sense and cognitive intentions. Consequently, among the researchers working within the fields of problem solving, information theory and artificial intelligence, there seems to be some agreement in the consideration that the creative process of the mind depends on the existence of certain patterns, or mind structure. Moreover, there is also some agreement on the fact that the mind can only be creative when it operates within a restricted territory or conceptual space. Both the notion of pattern and the notion of a conceptual space conform to the idea of morphological categorization held in the past by some architectural theorists.

It shows that the value of building pre-conceived formal notion can be seen in its application to design methods used to reference and make appropriate choice of a future design project. Using existing architectural language will also provide the architect with the much needed cognitive reference points which will elevate his or her understanding of the design problem though the use of mental, physical, or visual simulations.

Contemporary urban texture is experiencing many quality changes than ever. Heterogeneous cultural mix and composite substances are clearly re-organizing urban variables that have not yet showed a clear direction. Also, today's architecture is no longer subordinated within any urban context, but extremely dependent that architecture itself became a major variable. In other words, dealing with a simple form of any known shape will deliver its characteristic in a straightforward manner. However, today's architecture shows a strong scenographical shape based on visual dynamics and multifariousness sequence of continuing momentum.

Similarly, Glucksman and Danks (1968) support this idea that object classification into forms affects the public response, not only in the focus and memory of the object, but also the object's usage as well. Therefore, architects should understand that even though the Modern Movement endeavored to achieve the substantive modification of building forms, the basic architectural building image remained a force for cultural continuity (Robinson, 1994).

The most distinctive characteristic of contemporary architecture would be variety and diversity based on multifarious culture. Also, the major variable of any indeterminate architectural form would be visual dynamics that has relieved from modern uniformities. In other words, modern technology allows designers to eliminate rational orders with linear geometrical shape that used to have symbolic cultural meanings and compositions. Instead, curvilinear characteristics with extreme directed tension are providing unregulated patterns of indeterminate building forms based on personal intuitions and preferences.

However, this study would like to end this complicated argument by saying that commonly shared morphological notion can be understood as another term for building precedent that have necessarily been influenced by cultural and social factors that have evolved through times. Therefore, design approaches based on pre-conceived formal notion emphasis on the generation of new architectural form through reference to or adaptation of a vocabulary of pre-existing forms. In other words, one of the architect's promising design strategies is to engage in morphological thinking to create architecture that either accepts, adapts or transforms a particular building shape into a new identifiable form.

In summary, this study synthesized a wide range of literature review regarding building morphology and its related methodological notions in design process. This study was also conducted with a qualitative stance, which is an inductive way to come to the conclusion of the value of culturally gained image in architecture. However, the intention of this study is not to claim that a culturally known image must be the basis in architectural design in general because it would be difficult for an architect to ignore the contemporary trend in building form. Therefore, for further studies, it would be interesting to empirically evaluate variables that this inductive speculation provided regarding identifiable cultural image in today's free form architecture.

\section{REFERENCES}

Berman, B. (1988) From the New Criticism to Deconstruction. Urbana and Chicago: University of Illinois Press

Broadbent, G. (1973) Design in Architecture: Architecture and the Human Sciences. New Work: John Wiley and Sons

Cross, N. (1984) Developments in Design Methodology. New York: John Wiley and Sons

Galle, P. (1999) Design as Intentional Action: A Conceptual Analysis. Design Studies, 20(1), 57-81

Gelernter, M. (1995) Source of Architectural Form. Manchester: Manchester University Press

Jansson, DG., Condor SS. And Brock, HR (1993) cognition in Design: Viewing the Hidden Side of the Design Process. Environment Planning B: Planning and Design, 19(1), 23-29

Kaplan, S. and Kaplan, R. (1981) Cognitions and Environment: Functioning in an Uncertain World. New York: Praeger

Kelbaugh, D (1996) Typology - Architecture of Limits. Architectural Theory Review, 1(2), 33-52

Kneller, GF (1965) The Art and Science of Creativity. New York: Holt, Rinehart and Winston

Ladsun, D (1965) An Architect's Approach to Architecture. RIBA Journal, 72(April), 184-195

Lawson, B (1997) How Designers Think: The Design Process Demystified. London: The Architectural Press

Layder, D (1990) The Realist Image in Social Science. New York: St. Martin's Press

Mitchell, WJ (1990) The Logic of Architecture: Design, Computation and Cognition. Cambridge, MA: MIT Press

Nasar, J (1998) The Evaluative Image of the City. New York: John Wiley and Sons

Norman, D. (1993) Things that Make Us Smart: Defending Human Attributes in the Age of the Machine. Reading, MA: AddisonWesley Publishing Compane 
Quantrill, M. (1985) Reima Pietilä. New York: Rizzoli

Schön, D. (1988) Designing: Rules, Types and Works. Design Studies, 9(1), 23-29

Slife, B. (1995) What's Behind the Research. Thousand Oaks, CA: Sage

Smithies, KW (1981) Principles of Design in Architecture. New York: Van Nostrand Reinhold

(Date of Submission : 2011.6.26) 